\title{
CHARACTERISTIC PARAMETER VALUES FOR AN INTEGRAL EQUATION*
}

BY W. A. HURWITZ

1. Introduction; Hermitian Kernel. It is well known, though seldom explicitly mentioned, that the properties of real symmetric kernels hold, with only trivial alterations in the usual proofs, for complex Hermitian kernels. It is the purpose of this note to show that kernels of certain other kinds have analogous properties, with the role of the real axis as bearer of the characteristic parameter values taken by any other straight line in the plane.

All functions of $x, y, s$ which appear will be understood to be complex functions defined for real values of each of the variables in the closed interval $(a, b)$; all integrals will be taken between the limits $a, b$, which will not be written. We call $\lambda$ a characteristic parameter value (hereafter abbreviated cpv) for the kernel $K(x, y)$ if there exist nontrivial solutions of the equations

$$
\begin{aligned}
& u(x)=\lambda \int K(x, s) u(s) d s, \\
& v(y)=\lambda \int v(s) K(s, y) d s .
\end{aligned}
$$

The cpv's and the corresponding solutions of (1) (normalized and orthogonalized), arranged in the usual order, will sometimes be denoted by $\lambda_{p}, \varphi_{p}(x)[p=1,2, \ldots]$. A cpv is a pole (for at least some values of $x, y$ ) of the resolvent function $K(x, y ; \lambda)$, which is elsewhere analytic in $\lambda$. The resolvent satisfies the equation

(3) $K(x, y ; \lambda)-K(x, y ; \mu)=(\lambda-\mu) \int K(x, s ; \lambda) K(s, y ; \mu) d s$ whenever neither $\lambda$ nor $\mu$ is a cpv. Since $K(x, y ; 0)=K(x, y)$, (3) reduces for $\lambda=0$ and for $\mu=0$ to the simpler resolvent formulas used in the process of solving the Fredholm equation.

\footnotetext{
* Presented to the Society, December 30, 1924.
} 
A continuous* kernel $K(x, y)$ is closed if the equation $\int K(x, s) u(s) d s=0$ is satisfied by no continuous function except $u(x)=0$.

The properties of Hermitian kernels with which we are particularly concerned may be stated as follows:

If $K(x, y)$ is continuous and not identically zero, and $\dagger$ $\bar{K}(y, x)=K(x, y)$, then (I) every cpv is real; (II) every pole of the resolvent is of the first order; (III) there is at least one cpv; if $K$ is closed, the set of cpv's is infinite; (Iv) if for a cpv $\lambda, \varphi(x)$ is a solution of $(1)$, then $\bar{\varphi}(y)$ is a solution of (2); (v) the series $\$ \sum \varphi_{p}(x) \bar{\varphi}_{p}(y) / \lambda_{p}^{m}$ converges uniformly in $x, y$ and represents $K_{m}(x, y)$ for $m \geqq 2$; it represents. $K(x, y)$ for $m=1$ provided it converges uniformly; (vI) any function $f(x)$ expressible in the form $\int K(x, s) u(s) d s$, where $u(x)$ is continuous, can be expanded in the uniformly convergent series $f(x)=\sum \varphi_{p}(x) \int \bar{\varphi}_{p}(s) f(s) d s$.

2. Straight Line through Origin. Suppose that instead of the Hermitian condition we have $\bar{K}(y, x)=\zeta K(x, y)$, where $K$ is not identically zero; then

$$
\bar{K}(x, y)=\zeta K(y, x)=\zeta \bar{\zeta} \bar{K}(x, y),
$$

so that $|\zeta|=1$. Denote by $\sqrt{\zeta}$ either square root of $\zeta$, and let $L(x, y)=\sqrt{\zeta} K(x, y)$. If $\lambda$, $\mu$ denote corresponding cpv's for $K, L$, then $\lambda=\mu \sqrt{\zeta}$. Since

$$
\bar{L}(y, x)=\frac{1}{\sqrt{\zeta}} \bar{K}(y, x)=\sqrt{\zeta} K(x, y)=L(x, y),
$$

$L$ is Hermitian and $\mu$ is real. Thus $\lambda$ lies on a line through the origin and the point $\sqrt{\zeta}$. The other properties are readily verified, and we have the following result.

* The assumption of continuity is made for simplicity; the usual extensions, with the use of Lebesgue integrals, are possible.

$\dagger$ A bar over a symbol for a variable denotes the conjugate of that variable; a bar over a symbol for a function denotes the conjugate of the whole expression represented by the function; for instance, $\bar{K}(x, y ; \lambda)$ is an abbreviation for $\overline{K(x, y ; \lambda)}$.

$\ddagger$ If the number of cpv's is finite, the series terminates, and the "convergence" is uniform in $x, y$ for any value of $m$. 
If $K(x, y)$ is continuous and not identically zero, and $\bar{K}(y, x)=\zeta K(x, y)$, then $|\zeta|=1$; (I) every cpv is on the line through the origin and $\sqrt{\bar{\zeta}}$; (II-VI) the other properties stated in $\S 1$ hold without change.

A case of especial interest is that of the skew-Hermitian kernel: $\bar{K}(y, x)=-K(x, y)$; here $\zeta=-1$ and the cpv's are pure imaginary. Current treatises* prove the results of $\S 1$ only for real symmetric kernels, and are therefore able to deduce the corresponding facts for real skewsymmetric kernels only by repeating the proofs throughout; whereas the procedure here given makes the second theory essentially a mere special case of the first.

3. Special Straight Line not through Origin. Now suppose that $K$ is not identically zero and satisfies one of the relationst

$$
\begin{aligned}
K(x, y)+\bar{K}(y, x) & =2 \int K(x, s) \bar{K}(y, s) d s, \\
K(x, y)+\bar{K}(y, x) & =2 \int \bar{K}(s, x) K(s, y) d s
\end{aligned}
$$

for definiteness, say (4). By (3), if $K(x, y ; 2)$ exists,

$$
K(x, y ; 2)=K(x, y)+2 \int K(x, s) K(s, y ; 2) d s ;
$$

and, conversely, this relation is satisfied only by $K(x, y ; 2)$; hence (4) is equivalent to

$$
K(x, y ; 2)=-\bar{K}(y, x),
$$

and (5) holds also. Since $K$ has at most a countably infinite set of cpv's, there is on the line $R(\lambda)=1$ a point $\lambda=\lambda_{0}$ which is not a cpv; thus

(7) $K\left(x, y ; \lambda_{0}\right)=K(x, y)+\lambda_{0} \int K(x, s) K\left(s, y ; \lambda_{0}\right) d s$

* Lalesco, Introduction à la Théorie des Équations Intégrales, pp. 64, 73; Vivanti, Elementi della Teoria delle Equazione Integrali Lineari, pp. 207, 241.

$\dagger$ I omit the simple heuristic steps by which one is led to these equations as the natural generalization of the Hermitian condition. 
and

(8)

$$
\begin{aligned}
& K\left(x, y ; \lambda_{0}\right)=K(x, y ; 2) \\
& \quad+\left(\lambda_{0}-2\right) \int K\left(x, s ; \lambda_{0}\right) K(s, y ; 2) d s .
\end{aligned}
$$

From (8) we deduce, by interchanging $x, y$ and taking conjugates,

(9) $\bar{K}\left(y, x ; \lambda_{0}\right)=\bar{K}(y, x ; 2)$

$$
+\left(\bar{\lambda}_{0}-2\right) \int \bar{K}(s, x ; 2) \bar{K}\left(y, s ; \lambda_{0}\right) d s,
$$

or, by (6) and the relation $\lambda_{0}+\bar{\lambda}_{0}=2$,

(10) $\bar{K}\left(y, x ; \lambda_{0}\right)=-K(x, y)+\lambda_{0} \int K(x, s) \bar{K}\left(y, s ; \lambda_{0}\right) d s$.

Call $M(x, y)=K\left(x, y ; \lambda_{0}\right)+\bar{K}\left(y, x ; \lambda_{0}\right)$. By (7) and (10),

$$
M(x, y)=\lambda_{0} \int K(x, s) M(s, y) d s,
$$

so that $M(x, y)=0$ and

$$
\bar{K}\left(y, x ; \lambda_{0}\right)=-K\left(x, y ; \lambda_{0}\right) .
$$

If we write $N(x, y)=-K\left(x, y ; \lambda_{0}\right)$, and call $N(x, y ; \mu)$ the resolvent to $N(x, y)$, then $N\left(x, y ; \lambda-\lambda_{0}\right)=K(x, y ; \lambda)$; also

$$
\begin{aligned}
K(x, y) & =N(x, y)-\lambda_{0} \int K(x, s) N(s, y) d s, \\
& =N(x, y)-\lambda_{0} \int N(x, s) K(s, y) d s .
\end{aligned}
$$

By (11), $\bar{N}(y, x)=-N(x, y)$; and by $\S 2$, with $\zeta=-1$, any cpv $\mu$ for $N$ is a pure imaginary: $R(\mu)=0$. Thus a cpv $\lambda$ for $K$ must satisfy $R\left(\lambda-\lambda_{0}\right)=0$ or $R(\lambda)=1$.

This condition replaces (I) of $\S 1$. The other conditions may also be carried over; only (v), (vI), and the second part of (III) require any special consideration, which we now proceed to give.

In view of (12), it is readily seen that the equations

$$
f(x)=\int K(x, s) u(s) d s, \quad f(x)=\int N(x, s) v(s) d s
$$

are equivalent if $v(x)=u(x)-\lambda_{0} f(x)$; hence the hypothesis concerning $f$ in (v), involving $K$, may be stated equivalently 
involving in the same way $N$, for which by $\S 2$ the conclusion follows. Also by putting $f(x)=0$ in (13), we see that the hypothesis of the second part of (II) may be put in terms of $N$ instead of $K$.

This leaves only (vI) to be proved. By (vI) of $\S 2$ stated for $N$, with $\zeta=-1, m=2$, we have

$$
N_{2}(x, y)=\sum \frac{\varphi_{p}(x) \overline{\varphi_{p}}(y)}{\left(\lambda_{p}-\lambda_{0}\right)^{2}},
$$

the series converging uniformly in $x, y$. From (12),

$K_{2}(x, y)=N_{2}(x, y)-2 \lambda_{0} \int K(x, s) N_{2}(s, y) d s$

by means of which (14) yields

$$
+\lambda_{0}^{2} \int K_{2}(x, s) N_{2}(s, y) d s,
$$

$$
K_{2}(x, y)=\sum \frac{\varphi_{p}(x) \bar{\varphi}_{p}(y)}{\lambda_{p}^{2}},
$$

the series converging uniformly. Direct computation from (15) of the higher kernels gives the corresponding series for greater values of $m$. For $m=1$, suppose the series converges uniformly, and write

$$
A(x, y)=\frac{\varphi_{p}(x) \bar{\varphi}_{p}(y)}{\lambda_{p}} .
$$

By use of the relation

$$
\varphi_{p}(x)+\lambda_{0} \int N(x, s) \varphi_{p}(s) d s=\frac{\lambda_{p}}{\lambda_{p}-\lambda_{0}} \varphi_{p}(x),
$$

which is an immediate consequence of (12), we find

$$
A(x, y)+\lambda_{0} \int N(x, s) A(s, y) d s=\sum \frac{\varphi_{p}(x) \bar{\varphi}_{p}(y)}{\lambda_{p}-\lambda_{0}} .
$$

This series also converges uniformly and hence represents $N(x, y)$. Thus

$$
A(x, y)+\lambda_{0} \int N(x, s) A(s, y) d s=N(x, y)
$$

and $A(x, y)=K(x, y)$. 
All the expected results have now been shown to hold in the present case; I defer their formal statement until a further extension is made.

4. Straight Line not through Origin. More generally, let

$$
\bar{\alpha} K(x, y)+\alpha \bar{K}(y, x)=2 \cdot \int K(x, s) \vec{K}(y, s) d s
$$

or else

$$
\overline{\boldsymbol{\alpha}} K(x, y)+\alpha \bar{K}(y, x)=2 \int \bar{K}(s, x) K(s, y) d s,
$$

where $\alpha \neq 0$. Writing $K(x, y)=\alpha L(x, y)$, we see that $L$ satisfies the condition stated for $K$ in (4) or (5). Hence a $\operatorname{cpv} \mu$ for $L$ satisfies $R(\mu)=1$, and a $\operatorname{cpv} \lambda$ for $K$ satisfies $R(\alpha \lambda)=1$.

The verification of all the other results of $\S 3$ is easy. We have the following theorem.

If $K(x, y)$ is continuous and not identically zero, and satisfies one of the conditions

$$
\begin{aligned}
& \bar{\alpha} K(x, y)+\alpha \bar{K}(y, x)=2 \int K(x, s) \bar{K}(y, s) d s, \\
& \bar{\alpha} K(x, y)+\alpha \bar{K}(y, x)=2 \int \bar{K}(s, x) K(s, y) d s,
\end{aligned}
$$

where $\alpha \neq 0$, it satisfies the other also; (I) every cpv $\lambda$ is on the straight line $R(\alpha \lambda)=1$; (II-VI) the other properties stated in $\S 1$ hold without change.

5. Conclusion. We have given conditions on the kernel which will cause the cpv's to lie on any chosen line through the origin in $\S 2$, and on any line not through the origin in $\S 4$. The results of $\S 2$ are not contained in those of $\S 4$; but if we put $\alpha=r i / \sqrt{\zeta}$, where $|\zeta|=1, r$ is real and positive, we may view them as limiting cases for $r \rightarrow \infty$. A slight change of notation would of course make possible the inclusion of all the results in a single theorem.

Cornell University 